University of South Florida

DIGITAL COMMONS

Digital Commons @ University of

@ UNIVERSITY OF SOUTH FLORIDA

South Florida

1989

\title{
Effect of Rotation on Vertical Mixing and Associated Turbulence in Stratified Fluids
}

L. H. Kantha

\author{
A. Rosati \\ B. Galperin \\ Princeton University, bgalperin@usf.edu
}

Follow this and additional works at: https://digitalcommons.usf.edu/msc_facpub

Part of the Life Sciences Commons

\section{Scholar Commons Citation}

Kantha, L. H.; Rosati, A.; and Galperin, B., "Effect of Rotation on Vertical Mixing and Associated Turbulence in Stratified Fluids" (1989). Marine Science Faculty Publications. 1474.

https://digitalcommons.usf.edu/msc_facpub/1474

This Article is brought to you for free and open access by the College of Marine Science at Digital Commons @ University of South Florida. It has been accepted for inclusion in Marine Science Faculty Publications by an authorized administrator of Digital Commons @ University of South Florida. For more information, please contact digitalcommons@usf.edu. 


\title{
Effect of Rotation on Vertical Mixing and Associated Turbulence in Stratified Fluids
}

\author{
L. H. Kantha \\ Institute for Naval Oceanography, Stennis Space Center, Mississippi
}

A. Rosati

Geophysical Fluid Dynamics Laboratory, Princeton, New Jersey

B. GALPERIN

Department of Mechanical and Aerospace Engineering, Program in Applied and Computational Mathematics Princeton University, Princeton, New Jersey

\begin{abstract}
Combined effects of stratification and rotation on vertical mixing and the characteristics of associated small-scale turbulence are explored using second-moment closure methodology; the rotational terms in the equations for Reynolds stresses and turbulent heat fluxes are retained, not ignored as in earlier works. Semianalytical results valid for arbitrary values of rotation and stratification are derived by further invoking the local equilibrium limit of closure. Two cases are considered: nonzero vertical rotation and nonzero meridional rotation; the latter case is of more general interest in geophysics because of its potential application to equatorial mixed layers. In both cases the influence of rotation on mixing coefficients and Monin-Obukhov constant flux layer similarity relations is investigated for arbitrary values of rotation and stratification. In both cases, turbulent mixing coefficients assume tensorial properties. However, meridional rotation appears to have a stronger influence on vertical mixing and turbulence characteristics than does vertical rotation. These results, along with perturbation expansions for weak rotation, suggest that for geophysical flows, in most cases, the direct effect of rotation on vertical turbulent mixing itself is but a small correction, a few tens of percent at best. It is seldom large, although it might not be negligible in some particular cases. Nevertheless, the study of rotational effects on small-scale turbulence provides a fascinating insight into the direct impact of rotation on the characteristics of small-scale turbulence and mixing in stratified fluids; the results are also of interest in other fields such as engineering.
\end{abstract}

\section{INTRODUCTION}

Stratification is well known to strongly affect small-scale turbulence that is directly responsible for vertical mixing in geophysical flows, but rotational effects have traditionally been ignored as negligible. We do know that rotation exerts a profound influence on fluid flows, two excellent examples being geophysical flows and flows in turbomachinery. On a planet such as Earth, fluid motions at planetary scales are dominated by rotational effects. Therefore rotational terms have been routinely incorporated in the governing equations for mean flow quantities in these flows. On such large scales, the turbulence itself is strongly affected by Earth's rotation. Indeed, the study of geostrophic turbulence has been an important preoccupation of geophysicists. The reader is referred for example to the classical work on the subject by Rhines [1975, 1977, 1979].

However, vertical mixing in geophysical boundary layers is principally due to small-scale turbulence, with scales ranging from Kolmogorov scale at the lower end to a fraction of the vertical extent of these layers. Therefore there is a natural upper bound on turbulence scales responsible for vertical mixing. Since the effects of rotation can be expected to scale with the turbulence length scale, they have traditionally been assumed to be negligibly small as far as vertical mixing and small-scale turbulence are concerned. However,

Copyright 1989 by the American Geophysical Union.

Paper number $89 \mathrm{JC} 00025$.

0148-0227/89/89JC-00025\$05.00 the validity of this assumption has not been rigorously tested, and the effect of rotation on the small-scale turbulence field has received little attention so far. In a geophysical context, it was a common practice to ignore rotational terms in turbulence equations [e.g., Mellor and Yamada, 1982; Lewellen, 1977; Launder et al., 1975; Rodi, 1987], even though the need for investigating their effects has been acknowledged [Mellor and Yamada, 1982]. Only recently has attention been called to the potential importance of rotation on the small-scale turbulence itself [Garwood et al., $1985 a, b]$ in geophysics, although engineers have studied this aspect in the context of turbomachinery for quite some time (see, for example, the recent review by Lakshminarayana [1986]), because in high-speed turbomachinery and laboratory flows with background rotation, it is important to account for the effect of rotation both on the mean and turbulent flow.

It is our intent in this paper to explore the effects of rotation on small-scale turbulence and vertical mixing in the presence of strong gravitational stratification as appropriate to geophysical flows. As a corollary, we will investigate these effects on turbulence in neutrally stratified flows (of importance to engineers) as well. Such an investigation is a natural and logical extension of earlier works on vertical mixing and turbulence in geophysical boundary layers [e.g., Mellor, 1973; Lewellen, 1977; Launder et al., 1975; Mellor and Yamada, 1982]. We are interested, in particular, in quantifying the effect of rotation on small-scale turbulence in the geophysical context. In pursuit of this goal, we will 
explore analytically tractable solutions in the limit of local equilibrium and cite numerical solutions in the more complex situations. We will retain the rotational terms in the equations for the second moments; this does introduce algebraic complexity, as we will see later.

Large-scale turbulence is obviously strongly affected by rotation. There is also no doubt as to whether small-scale turbulence is affected by strong rotation. Experimental evidence on this in neutral flows is rather convincing. For example, experiments by Johnston et al. [1972] on a duct flow with spanwise rotation showed that rotation damps turbulence on the leading side and enhances it on the trailing side. More recently, Koyama et al. [1979] and Watmuffet al. [1985] have also shown that strong rotation has a profound effect on turbulence in boundary layers. Therefore engineers dealing with fluid flows in rapidly rotating devices such as turbomachinery have always been concerned with rotational effects on turbulence (see the review by Bradshaw [1973]), but only recently have they attempted a systematic study of these effects using higher-order closure techniques (see the recent review by Lakshminarayana [1986]; see also Galperin and Kantha [1989]).

Experiments on turbulence generated by an oscillating grid in a rotating tank by Hopfinger and his colleagues [Hopfinger et al., 1983; Hopfinger, 1987] have also shown that far away from the generating grid, where rotational effects become stronger, the nature of turbulence changes drastically, and turbulence no longer has its characteristic three-dimensional structure. Experiments on turbulence generated in a wind tunnel by a rotating grid show the rather subtle effect of rotation on turbulence [Wigeland and Nagib, 1978]. Rotation appears to slow down the rate of turbulence decay! Bardina et al. [1985] have also shown a similar effect in their numerical simulation of isotropic turbulence using direct computational techniques.

On the other hand, there appear to be no laboratory experiments on the combined effects of rotation and stratification on turbulence, and observational data have not been carefully examined to discern the effects of rotation on geophysical shear layers and turbulence in them. In these flows, stratification exerts an overwhelming influence, and geophysicists have naturally concentrated their attention on that. However, Garwood et al. [1985a, b] have recently questioned the wisdom of neglecting rotational effects on small-scale geophysical turbulence. Their study suggests that at least in some situations, rotational effects cannot be ignored. They postulate that it is because of the influence of the horizontal component of Earth's rotation that the mixed layer in the equatorial Pacific is deeper on the western side. Their reasoning is, however, based on a series of assumptions, whose validity is not beyond any question. A systematic study of the combined effects of stratification and rotation on geophysical mixing, from fundamental considerations that would show conclusively the rotational influence, has so far not been undertaken. It is our objective to do so, using well-known second-moment closure techniques.

This effort was undertaken in parallel with the study of Galperin et al. [1989], which uses a one-dimensional numerical ocean model that involves the solution of prognostic equations for turbulence quantities. While that study concentrates on numerical solutions for near-equatorial oceanic mixed layers, this study aims to derive semianalytical results in the limit of local equilibrium that are amenable to more straightforward interpretation. While both approaches are valid for arbitrary rotation and stratification, this study does not involve explicit consideration of the turbulence length scale. On the other hand, this approach uses a simplified turbulence model for reasons of analytical tractability, whereas the turbulence model of Galperin et al. [1989] is more general in that it retains diffusion, advection, and tendency terms.

The reader is referred to Galperin et al. [1989] and Galperin and Kantha [1989] for detailed discussions of the effect of rotation on neutrally stratified flows, for which there do exist some laboratory data. The focus of the latter paper is the rotational effect on turbulence in flow machinery of engineering interest, while a substantial portion of the former is devoted to rotational effects on turbulence in unstratified geophysical boundary layers.

\section{Governing Equations}

The governing equations for mean quantities under Boussinesq approximation can be written in their component form as

$$
\begin{gathered}
\frac{D U}{D t}+f_{y} W-f V=-\frac{\partial P}{\partial x}+\frac{\partial \tau_{x}}{\partial z} \\
\frac{D V}{D t}+f U=-\frac{\partial P}{\partial y}+\frac{\partial \tau_{y}}{\partial z} \\
\frac{D W}{D t}-f_{y} U=-\frac{\partial P}{\partial z}-g \frac{\rho}{\rho_{0}} \\
\frac{\partial U}{\partial x}+\frac{\partial V}{\partial y}+\frac{\partial W}{\partial z}=0 \\
\frac{D \Theta}{D t}=\frac{\partial Q}{\partial z}
\end{gathered}
$$

The velocities in the zonal $(x)$, meridional $(y)$, and vertical $(z)$ directions are $U, V$, and $W ; \tau_{x}$ and $\tau_{y}$ are the kinematic shear stresses in the zonal and meridional directions; $Q$ is the heat flux; $\rho, P$, and $\Theta$ are density, kinematic pressure, and potential temperature; and $f$ and $f_{y}$ are the components of the Coriolis term (twice the angular rotation of Earth) in the vertical and meridional directions $\left(f_{x}\right.$ is zero). Time is denoted by $t$.

For geophysical shear layers, because the scales in the horizontal are much larger than those in the vertical $(\partial / \partial z \gg$ $\partial / \partial y)$, it follows that

$$
\boldsymbol{W} \ll U, V
$$

Therefore the term $f_{y} W$ in (1) can be ignored, as has been done traditionally. Terms containing $f$ in (1) and (2) are important in geophysical flows and, of course, cannot be ignored.

It is also the usual practice to neglect the acceleration terms in (3): the so-called hydrostatic approximation. However, when $f_{y} \neq 0$, the accuracy of this assumption is somewhat less (even though it is still valid in most situations) because the rotational term, $f_{y} U$, is still 2 orders of magnitude less than the dominant buoyancy term, $g \rho / \rho_{0}$. (For the atmosphere, typical values might be $f_{y} \sim 10^{-4} \mathrm{~s}^{-1}, U \sim 10 \mathrm{~m}$ $s^{-1}$, and $\rho / \rho_{0} \sim 0.02$.) Also, for consistency in energetics it is necessary to neglect (or retain) $f_{y}$ terms in both (1) and (3). Equation (5) does not contain any rotational terms. Thus 
only the vertical component of rotation, $f$, enters the equations for mean momentum. In contrast, both components appear in the equations for the turbulent (Reynolds) stresses and heat fluxes. However, no explicit rotational terms appear in the turbulence $\mathrm{KE}$ (kinetic energy) equation

$$
\frac{D}{D t}\left(\frac{q^{2}}{2}\right)-\frac{\partial}{\partial x_{k}}\left[q l S_{q} \frac{\partial}{\partial x_{k}}\left(\frac{q^{2}}{2}\right)\right]=P_{s}+P_{b}-\varepsilon
$$

and traditionally, rotational terms have also been ignored in the length scale equation. However, it may be appropriate to include rotational terms in the equation for turbulence macroscale [Bardina et al., 1985], which can then be written as

$$
\begin{aligned}
& \frac{D}{D t}\left(q^{2} l\right)-\frac{\partial}{\partial x_{k}}\left[q l S_{l} \frac{\partial}{\partial x_{k}}\left(q^{2} l\right)\right]=l E_{1}\left(P_{s}+E_{3} P_{b}\right) \\
&-E_{4} l \varepsilon\left[1+E_{2}\left(\frac{l}{k L}\right)^{2}\right]+E_{5}\left(f_{k} f_{k}\right)^{1 / 2} q^{2} l
\end{aligned}
$$

where the shear production $\boldsymbol{P}_{s}$, the buoyancy production $\boldsymbol{P}_{b}$, and dissipation $\varepsilon$ are given by

$$
\begin{gathered}
P_{s}=-\left\langle u_{i} u_{j}\right\rangle \frac{\partial U_{i}}{\partial x_{j}} \\
P_{b}=-\beta g_{i}\left\langle u_{i} \theta\right\rangle \\
\varepsilon=\frac{q^{3}}{B_{1} l}
\end{gathered}
$$

and $f_{k}$ is the rotational vector equal to $\left(0, f_{y}, f\right)$ in geophysics. The constants $E_{1}$ to $E_{5}$ are defined below.

The rotational term in (8) follows from the studies of grid-generated turbulence under rotation [see Bardina et al., 1985] although Launder et al. [1987] suggest that its effect on their simulation of a rotating channel flow was not significant. Besides, empirical evidence for this term is still rather scanty, and rotational influence on dissipation rate needs to be explored further. Therefore the influence of rotation on turbulence length scale is as yet not fully understood and will doubtless be the subject of future research. Nevertheless, the term is included here for completeness, although in our subsequent analysis, the length scale equation does not enter, and therefore explicit effect of rotation on length scale does not matter.

The governing equations for the second moments, $\left\langle u_{1} u_{\rangle}\right\rangle$, $\left\langle u_{j} \theta\right\rangle$, and $\left\langle\theta^{2}\right\rangle$, can be simplified following the expansion scheme of Mellor and Yamada [1974], resulting in the so-called level $2 \frac{1}{2}$ model for turbulent mixing [Mellor and Yamada, 1982]. Using a slight modification of this expansion scheme [Galperin et al., 1988] results in a somewhat simpler set of algebraic equations for the second moments:

$$
\begin{aligned}
\left\langle u_{i} u_{j}\right\rangle= & \delta_{i j} \frac{q^{2}}{3}-\frac{3 A_{1} l}{q}\left[\left\langle u_{i} u_{k}\right\rangle \frac{\partial U_{j}}{\partial x_{k}}+\left\langle u_{j} u_{k}\right\rangle \frac{\partial U_{i}}{\partial x_{k}}\right. \\
& +\beta g_{j}\left\langle u_{i} \theta\right\rangle+\beta g_{i}\left\langle u_{j} \theta\right\rangle-C_{1} q^{2}\left(\frac{\partial U_{i}}{\partial x_{j}}+\frac{\partial U_{j}}{\partial x_{i}}\right) \\
& \left.+\frac{2}{3} \delta_{i j} \varepsilon+A_{3} f_{k}\left(\varepsilon_{j k l}\left\langle u_{l} u_{i}\right\rangle+\varepsilon_{i k l}\left\langle u_{l} u_{j}\right\rangle\right)\right]
\end{aligned}
$$

$$
\begin{aligned}
\left\langle u_{j} \theta\right\rangle=-\frac{3 A_{2} l}{q}\left[\left\langle u_{j} u_{k}\right\rangle \frac{\partial \Theta}{\partial x_{k}}\right. & +\left\langle\theta u_{k}\right\rangle \frac{\partial U_{j}}{\partial x_{k}} \\
& \left.+\beta g_{j}\left\langle\theta^{2}\right\rangle+A_{4} f_{k} \varepsilon_{j k l}\left\langle u_{l} \theta\right\rangle\right] \\
\left\langle\theta^{2}\right\rangle= & -\frac{B_{2} l}{q}\left\langle u_{k} \theta\right\rangle \frac{\partial \Theta}{\partial x_{k}}
\end{aligned}
$$

If only the explicit rotational terms are retained in the equations for Reynolds stresses and heat fluxes, then $A_{3}=$ $A_{4}=1$. Note that in this case, there is no modeling whatsoever as far as rotational terms are concerned (unlike for example, dissipation terms). This is a decided advantage, as it removes the modeling aspect from the picture and therefore renders the influence of rotation less ambiguous and the results more easily interpreted. However, rotation may influence the modeling of terms, in second-moment equations, that involve pressure fluctuations, namely, the pressure-strain covariance and $\left\langle p \partial \theta / \partial x_{\jmath}\right\rangle$ term. The model for the pressure-strain covariance involves two terms, one being the return to isotropy (Rotta hypothesis) and the other accounting for rapid distortion pressure effects (see Mellor and Yamada [1982] and Rodi [1987], for example). It can be shown [Zeman and Tennekes, 1975] that the rotational influence on these terms can be modeled using terms, containing the rotational vector, whose form is identical to that of the explicit rotational terms in the second-moment equations (see also Galperin et al. [1989]). Thus the only change in (10) and (11) that results from including both the implicit and explicit rotational terms is that the values of coefficients $\boldsymbol{A}_{3}$ and $\boldsymbol{A}_{4}$ are no longer unity. However, there is at present no reliable empirical information as to the likely value of $A_{3}$ or $A_{4}$. There are some indications that $A_{3}$ is somewhat less than unity, and the implicit terms tend to counteract the explicit ones [Zeman and Tennekes, 1975]. However, $A_{3}$ is not much different from 1 , and equating it to 1 perhaps overestimates slightly the influence of rotation on turbulence. In the following analysis, we will put $A_{3}=1$, but it is easy enough to modify the results, once incontrovertible evidence becomes available, just by replacing $f_{k}$ by $A_{3} f_{k}$ in the Reynolds stress and by $A_{4} f_{k}$ in the heat flux equations. Equations (7), (8), and (10)-(12) can be simplified for shear layers by taking advantage of the fact that the length scale in one direction is much smaller than those in the other directions. For geophysical shear layers, $\partial / \partial z \gg \partial / \partial x, \partial / \partial y$, where $z$ is the vertical direction. Also $g_{1}=g_{2}=0$ and $g_{3}=$ $-g$, so that

$$
\begin{gathered}
P_{s}=-\langle u w\rangle \frac{\partial U}{\partial z}-\langle v w\rangle \frac{\partial V}{\partial z} \\
P_{b}=\beta g\langle w \theta\rangle
\end{gathered}
$$

The equations for the second moment turbulence quantities can therefore be written, in their component form, as

$$
\begin{aligned}
\left\langle u^{2}\right\rangle=\frac{q^{2}}{3}\left(1-6 \frac{A_{1}}{B_{1}}\right)-\frac{6 A_{1} l}{q}\langle u w\rangle & \frac{\partial U}{\partial z} \\
+ & \frac{6 A_{1} l}{q}\left(f\langle u v\rangle-f_{y}\langle u w\rangle\right.
\end{aligned}
$$




$$
\begin{aligned}
& \left\langle v^{2}\right\rangle=\frac{q^{2}}{3}\left(1-6 \frac{A_{1}}{B_{1}}\right)-\frac{6 A_{1} l}{q}\langle v w\rangle \frac{\partial V}{\partial z}-\frac{6 A_{1} l}{q} f(u v) \\
& \left\langle w^{2}\right\rangle=\frac{q^{2}}{3}\left(1-6 \frac{A_{1}}{B_{1}}\right)+\frac{6 A_{1} l}{q} \beta g\langle w \theta\rangle+\frac{6 A_{1} l}{q} f_{y}\langle u w\rangle \\
& \langle u v\rangle=\frac{3 A_{1} l}{q}\left[-\langle u w\rangle \frac{\partial V}{\partial z}-\langle v w\rangle \frac{\partial U}{\partial z}-f_{y}\langle v w\rangle-f\left(\left\langle u^{2}\right\rangle-\left\langle v^{2}\right\rangle\right)\right] \\
& \langle u w\rangle=\frac{3 A_{1} l}{q}\left[-\left(\left\langle w^{2}\right\rangle-C_{1} q^{2}\right) \frac{\partial U}{\partial z}+\beta g\langle u \theta\rangle\right. \\
& \left.+f\langle v w\rangle-f_{y}\left(\left\langle w^{2}\right\rangle-\left\langle u^{2}\right\rangle\right)\right] \\
& \langle v w\rangle=\frac{3 A_{1} l}{q}\left[-\left(\left\langle w^{2}\right\rangle-C_{1} q^{2}\right) \frac{\partial V}{\partial z}\right. \\
& \left.+\beta g\langle v \theta\rangle-f\langle u w\rangle+f_{y}\langle u v\rangle\right] \\
& \langle u \theta\rangle=\frac{3 A_{2} l}{q}\left[-\langle u w\rangle \frac{\partial \Theta}{\partial z}-\langle w \theta\rangle \frac{\partial U}{\partial z}+f\langle v \theta\rangle-f_{y}\langle w \theta\rangle\right] \\
& \langle v \theta\rangle=\frac{3 A_{2} l}{q}\left[-\langle v w\rangle \frac{\partial \Theta}{\partial z}-\langle w \theta\rangle \frac{\partial V}{\partial z}-f\langle u \theta\rangle\right] \\
& \langle w \theta\rangle=\frac{3 A_{2} l}{q}\left[-\left\langle w^{2}\right\rangle \frac{\partial \Theta}{\partial z}+\beta g\left\langle\theta^{2}\right\rangle+f_{y}\langle u \theta]\right. \\
& \left\langle\theta^{2}\right\rangle=-\frac{B_{2} l}{q}\langle w \theta\rangle \frac{\partial \Theta}{\partial z}
\end{aligned}
$$

Constants, $A_{1}, A_{2}, B_{1}, B_{2}, C_{1}, E_{1}, E_{2}, E_{3}, E_{4}, E_{5}, S_{4}$ and $S_{l}$ are $0.92,0.74,16.6,10.1,0.08,1.8,1.33,1.0,1.0,0.04,0.2$ and 0.2 [Mellor and Yamada, 1982] and are assumed to be invariant. Note that the rotational terms redistribute energy among the three components of turbulence KE but do not change it, at least not directly (equations (7) and (14)-(15)). However, they do affect the Reynolds shear stress and heat flux terms (equations (17)-(22)) and therefore affect the production terms in (7) and therefore indirectly modify the turbulence KE. It is worth pointing out that it is essential to consider the entire set of equations (14)-(23), since rotational terms affect all the Reynolds stress and heat flux components. Considering only a subset of these equations (following Garwood et al. [1985]) is not self-consistent.

The presence of $f$ and $f_{y}$ in (14)-(22) imparts tensorial properties to the mixing coefficient for momentum (equation (25); see Galperin and Kantha [1989] and Galperin et al. [1989]) and makes it harder to derive algebraic expressions for the mixing coefficients. Nevertheless, it is possible to derive a set of three equations for the three quantities, $S_{M U}$, $S_{M V}$, and $S_{H}$ :

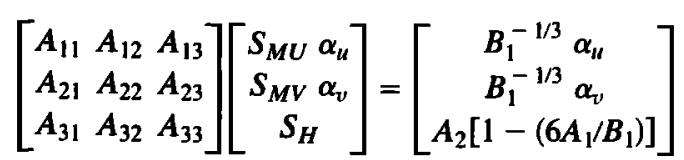

where

$$
S_{M U}=\frac{-\langle u w\rangle}{l q(\partial U / \partial z)}
$$

$$
\begin{gathered}
S_{M V}=\frac{-\langle v w\rangle}{l q(\partial V / \partial z)} \\
S_{H}=\frac{-\langle w \theta\rangle}{l q(\partial \Theta / \partial z)}
\end{gathered}
$$

Mixing coefficients for momentum and heat are $K_{M U}=$ $l q S_{M U}, K_{M V}=l q S_{M V}$, and $K_{H}=l q S_{H}$. The coefficients $A_{11}-A_{33}$ are given by

$A_{11}=1-9 A_{1} A_{2} G_{H}$

$$
\begin{gathered}
+36 A_{1}^{2} R o_{y}\left(\alpha_{u}+R o_{y}\right)+\frac{81 A_{1} A_{2}^{3} R o^{2} G_{H}}{1+9 A_{2}^{2} R o^{2}} \\
+\frac{54 A_{1}^{3} R o R o_{y}}{1+36 A_{1}^{2} R o^{2}}\left[\alpha_{v}-6 A_{1} R o\left(\alpha_{u}+R o_{y}\right)\right] \\
A_{12}=-3 A_{1} R o-\frac{27 A_{1} A_{2}^{2} R o G_{H}}{1+9 A_{2}^{2} R o^{2}} \\
+\frac{54 A_{1}^{3} R o R o_{y}}{1+36 A_{1}^{2} R o^{2}}\left(\alpha_{u}+R o_{y}+6 A_{1} R o \alpha_{v}\right) \\
A_{13}=-18 A_{1}^{2} G_{H} \alpha_{u}-9 A_{1} A_{2} G_{H}\left(\alpha_{u}+R o_{y}\right)-18 A_{1}^{2} G_{H} R o_{y} \\
-\frac{27 A_{1} A_{2}^{2} R o G_{H}}{1+9 A_{2}^{2} R o^{2}}\left[\alpha_{v}-3 A_{2} R o\left(\alpha_{u}+R o_{y}\right)\right] \\
A_{21}=18 A_{1}^{2} R o_{y} \alpha_{v}+3 A_{1} R o+\frac{27 A_{1} A_{2}^{2} R o G_{H}}{1+9 A_{2}^{2} R o^{2}}+\frac{9 A_{1}^{2} R o_{y}}{1+36 A_{1}^{2} R o^{2}} \\
\cdot\left[\alpha_{v}-6 A_{1} R o\left(\alpha_{u}+R o_{y}\right)\right]
\end{gathered}
$$

$$
\begin{aligned}
A_{22}=1-\frac{9 A_{1} A_{2} G_{H}}{1+9 A_{2}^{2} R o^{2}}+\frac{9 A_{1}^{2} R o_{y}}{1+36 A_{1}^{2} R o^{2}} \\
\cdot\left[\alpha_{u}+R o_{y}+6 A_{1} R o \alpha_{v}\right]
\end{aligned}
$$

$A_{23}=-18 A_{1}^{2} G_{H} \alpha_{v}-\frac{9 A_{1} A_{2} G_{H}}{1+9 A_{2}^{2} R o^{2}}$

$$
\begin{gathered}
\cdot\left[\alpha_{v}-3 A_{2} R o\left(\alpha_{u}+R o_{y}\right)\right] \\
A_{31}=18 A_{1} A_{2} R o_{y}+9 A_{2}^{2} R o_{y}-\frac{81 A_{2}^{4} R o^{2} R o_{y}}{1+9 A_{2}^{2} R o^{2}} \\
A_{32}=\frac{27 A_{2}^{3} R o R o_{y}}{1+9 A_{2}^{2} R o^{2}}
\end{gathered}
$$$$
A_{33}=1-18 A_{1} A_{2} G_{H}-3 A_{2} B_{2} G_{H}+9 A_{2}^{2} R o_{y}\left(\alpha_{u}+R o_{y}\right)
$$$$
+\frac{27 A_{2}^{3} R o R o_{y}}{1+9 A_{2}^{2} R o^{2}}\left[\alpha_{v}-3 A_{2} \operatorname{Ro}\left(\alpha_{u}+R o_{y}\right)\right]
$$

The quantities $R o$ and $R o_{y}$ are inverse Rossby numbers defined by

$$
\begin{gathered}
R o=\frac{f l}{q} \\
R o_{y}=\frac{f_{y} l}{q}
\end{gathered}
$$


The quantities $\alpha_{u}$ and $\alpha_{v}$ denote components of nondimensional shear

$$
\begin{gathered}
\alpha_{u}=\frac{l}{q} \frac{\partial U}{\partial z} \\
\alpha_{v}=\frac{l}{q} \frac{\partial V}{\partial z} \\
G_{M}=\alpha_{u}^{2}+\alpha_{v}^{2} \\
G_{H}=-\frac{l^{2}}{q^{2}} \beta g \frac{\partial \Theta}{\partial z}
\end{gathered}
$$

Note that unlike the case of $f_{k}=0, S_{M U}$ and $S_{M V}$ are not necessarily equal. Under the assumption of local equilibrium (level 2), the left-hand side of (7) can be neglected, and (7) can be written as

$$
S_{M U} \alpha_{u}^{2}+S_{M V} \alpha_{v}^{2}+S_{H} G_{H}=B_{1}^{-1}
$$

If we use the level $2 \frac{1}{2}$ equations of Mellor and Yamada [1982] instead, it is easy to show that the coefficients $A_{1}-A_{33}$ are replaced by $A_{11}^{1}-A_{33}^{1}$ in (24), where

$$
\begin{aligned}
A_{11}^{1}=A_{11}+6 A_{1}^{2} \alpha_{u}^{2}, & A_{12}^{1}=A_{12} \\
& +6 A_{1}^{2} \alpha_{u} \alpha_{u}, A_{13}^{1}=A_{13}+6 A_{1}^{2} G_{H} \alpha_{u} \\
A_{21}^{1}=A_{21}+6 A_{1}^{2} \alpha_{u} \alpha_{v}, & A_{22}^{1}=A_{22} \\
& +6 A_{1}^{2} \alpha_{v}^{2}, A_{23}^{1}=A_{23}+6 A_{1}^{2} G_{H} \alpha_{v} \\
A_{31}^{1}=A_{31}+6 A_{1} A_{2} \alpha_{u}, & A_{32}^{1}=A_{32} \\
& +6 A_{1} A_{2} \alpha_{v}, A_{33}^{1}=A_{33}+6 A_{1} A_{2} G_{H}
\end{aligned}
$$

and the right-hand side of (24) is replaced by

$$
\left[\begin{array}{c}
A_{1}\left(1-3 C_{1}\right) \alpha_{u} \\
A_{1}\left(1-3 C_{1}\right) \alpha_{v} \\
A_{2}
\end{array}\right]
$$

Equations (7) and (8) along with (24) can be solved numerically for arbitrary values of $R o$ and $R o_{y}$ [see Galperin et al., 1989]. Equation (24) can be solved rather efficiently using Cramer's rule for inverting matrices (the matrix dimension is only $3 \times 3$ ).

When $R o=R o_{y}=0$, equations (24) to (34) yield the modified $2 \frac{1}{2}$ level model results of Galperin et al. [1988] for $S_{M U}=S_{M V}$ $=S_{M}$ and $S_{H}$ (their equations 24 and 25). It can be shown that for the level $2 \frac{1}{2}$ approximation, we recover equations (34) and (35) of Mellor and Yamada [1982]. If in addition, we appeal to their equation (34) and invoke the local equilibrium limit, it can be shown that both of these approximations yield (41) of Mellor and Yamada [1982]. In this limit, turbulence production and dissipation are assumed to balance each other, and the diffusion, advection, and tendency terms are ignored in the equation for the second moments. This approximate balance between production and dissipation often prevails in geophysical boundary layers [see Mellor, 1973] and the local equilibrium limit is therefore useful, especially since it reduces the differential equations to algebraic equations and allows for semianalytical solutions.

In general, the limit of local equilibrium is also useful when the rotational effects, i.e., the inverse Rossby numbers $R o$ and
$R o_{y}$, are nonzero. However, the algebra is rather messy for the general case. We will therefore deal separately with two cases, the first one where $R o_{y}=0$ but $R o \neq 0$ and the second where $R o=0$ but $R o_{y} \neq 0$. By dealing with the local equilibrium limit in these subcases, it is our hope to be able to quantify rotational effects and investigate their importance or lack thereof in geophysical boundary layers, which are strongly dominated by stratification effects. As a corollary we will also derive results for the case of strong rotation but weak or no stratification, a situation of interest to engineers.

An attractive feature of this approach is that while in the approach of Galperin et al. [1989], the turbulence macroscale has to be predicted explicitly, in the present approach this is not necessary; therefore analytical results can be obtained, and to some extent a more straightforward interpretation can be made of the rotational influence on turbulence.

\section{Case of Zero Meridional, Nonzero Vertical ROTATION $\left(R o_{y}=0, R o \neq 0\right)$}

In this case, (24) can be written in complex notation as

$$
\begin{aligned}
S_{C}[(1 & \left.-\frac{9 A_{1} A_{2}}{1+9 A_{2}^{2} R o^{2}} G_{H}\right) \\
& \left.+i 3 A_{1} R o\left(1+\frac{9 A_{2}^{2}}{1+9 A_{2}^{2} R o^{2}} G_{H}\right)\right] \\
& -S_{H}\left[2 A_{1}+\frac{A_{2}}{1+9 A_{2}^{2} R o^{2}}\left(1-i 3 A_{2} R o\right)\right] \\
& 9 A_{1} G_{H} \alpha_{C}=B_{1}^{-1 / 3} \alpha_{C} \\
& S_{H}\left[1-3 A_{2}\left(6 A_{1}+B_{2}\right) G_{H}\right]=A_{2}\left(1-\frac{6 A_{1}}{B_{1}}\right)
\end{aligned}
$$

while (39) becomes

$$
\operatorname{Re}\left[S_{C} \alpha_{C}^{*}\right]+S_{H} G_{H}=B_{1}^{-1}
$$

where

$$
\begin{gathered}
S_{C}=S_{M U} \alpha_{u}+i S_{M v} \alpha_{v} \\
\alpha_{C}=\alpha_{u}+i \alpha_{v}
\end{gathered}
$$

and $\alpha_{C}^{*}$ is the complex conjugate of $\alpha_{C}$. Because of the absence of $f_{y}\left(R o_{y}=0\right)$, there is no loss of generality in aligning the $x$ coordinate in the direction of the local stress. Then by definition, $S_{M V}=0$, and algebra is greatly simplified.

\subsection{Mixing Coefficients}

In this section we would like to investigate the effect of $R o$ on mixing coefficients $S_{M U}$ and $S_{H}\left(S_{M V}=0\right.$ by definition). If we define $R i_{R}$ by

$$
R i_{R}=f / \frac{\partial U}{\partial z}
$$

noting that because $\langle v w\rangle=0, R i_{f}$ is given by

$$
R i_{f}=\frac{-\beta g\langle w \theta\rangle}{-(u w\rangle(\partial U / \partial z)}
$$

and substituting $q_{*}=q / u_{*}$, where $u_{*}^{2}=-\langle u w\rangle$, equations (42)-(46) can be reduced to 


$$
\begin{gathered}
\left(D_{R}^{2}+D_{I}^{2}\right) q_{*}^{3}-B_{1}\left(1-R i_{f}\right)\left(N_{R} D_{R}+N_{l} D_{l}\right)=0 \\
S_{M U}=B_{1}\left(1-R i_{f}\right) / q_{*}^{4} \\
S_{H}=A_{2}\left(1-\frac{6 A_{1}}{B_{1}}\right)-\frac{3 A_{2}\left(6 A_{1}+B_{2}\right)}{B_{1}\left(1-R i_{f}\right)} R i_{f}
\end{gathered}
$$

where

$$
\begin{gathered}
D_{I}=\frac{27 A_{1} A_{2}^{2} R i_{R} R i_{f}}{B_{1}^{2}\left(1-R i_{f}\right)^{2}} q_{*}^{4} \\
N_{I}=\frac{3 A_{1} R i_{R} q_{*}^{7}}{B_{1}^{3}\left(1-R i_{f}\right)^{3} S_{H}}\left[\frac{B_{1}^{2}\left(1-R i_{f}\right)^{2} S_{H}}{q_{*}^{4}}\right. \\
\left.+9 A_{2}^{2}\left(S_{H} R i_{R}^{2}-\frac{B_{1} R i_{f}\left(1-R i_{f}\right)}{q_{*}^{4}}\right)\right] \\
N_{R}=\frac{q_{*}^{5}}{B_{1}^{2}\left(1-R i_{f}\right)^{2} S_{H}}\left[\frac{B_{1}^{2}\left(1-R i_{f}\right)^{2} S_{H}}{q_{*}^{4}}\right. \\
\left.+\frac{9 A_{1} A_{2} B_{1}\left(1-R i_{f}\right) R i_{f}}{q_{*}^{4}}+9 A_{2}^{2} R i_{R}^{2} S_{H}\right] \\
D_{R}=\frac{B_{1}^{-1 / 3} q_{*}^{4}}{B_{1}^{2}\left(1-R i_{f}\right)^{2}}\left[\frac{B_{1}^{2}\left(1-R i_{f}\right)^{2}}{q_{*}^{2}}+9 A_{2}^{2} R i_{R}^{2}\right] \\
-\frac{9 A_{1} R i_{f} q_{*}^{6}}{B_{1}^{3}\left(1-R i_{f}\right)^{3}}\left[\left(2 A_{1}+A_{2}\right) \frac{B_{1}^{2}\left(1-R i_{f}\right)^{2}}{q_{*}^{4}}+9 A_{2}^{2} R i_{R}^{2}\right]
\end{gathered}
$$

The set of equations (49)-(55) can be solved readily by Newton-Raphson technique for coupled nonlinear equations. Figure 1 shows $S_{M U}$ and $S_{H}$ as functions of $R i_{f}$ for various values of $R i_{R} . S_{H}$ is not affected by rotation and therefore is a function not of $R i_{R}$, but only of $R i_{f}$. Figure 1 shows that the stratification flux Richardson number $R i_{f}$ for extinction of turbulence is a function of the magnitude of the rotational Richardson number $R i_{R}$. At $\left|R i_{R}\right|=1.5$, the extinction $R i_{f}$ is nearly zero, and at 0.75 it is about 0.1 , about half the value for zero rotation.

We note that in (49)-(55), $R i_{R}$ occurs only in powers of 2 or more; there are no terms linear in $R i_{f}$. This means that to $O\left(R i_{R}^{2}\right)$ the momentum mixing coefficient $S_{M U}$ is not affected by nonzero $f$.

It is also worth noting that even though $S_{M V}=0, \partial V / \partial z$ is nonzero and is in fact given by

$$
\frac{\partial V}{\partial z}=\left[\frac{N_{l} D_{R}-N_{R} D_{I}}{N_{R} D_{R}+N_{l} D_{I}}\right] \frac{\partial U}{\partial z}
$$

For $R i_{R}=0, N_{I}=D_{l}=0$; also $\partial V / \partial z=0$, and we recover zero rotation values for mixing coefficients. On the other hand, for $R i_{f}=0$, i.e., for neutral stratification, $D_{l}=0$ and

$$
\begin{gathered}
S_{M U}=B_{1}^{-1 / 3} \quad q_{*}=B_{1}^{1 / 3} \quad S_{H}=A_{2}\left(1-\frac{6 A_{1}}{B_{1}}\right) \\
\frac{\partial V}{\partial z}=3 A_{1} R o \quad R o=B_{1}^{-1 / 3} R i_{R}
\end{gathered}
$$
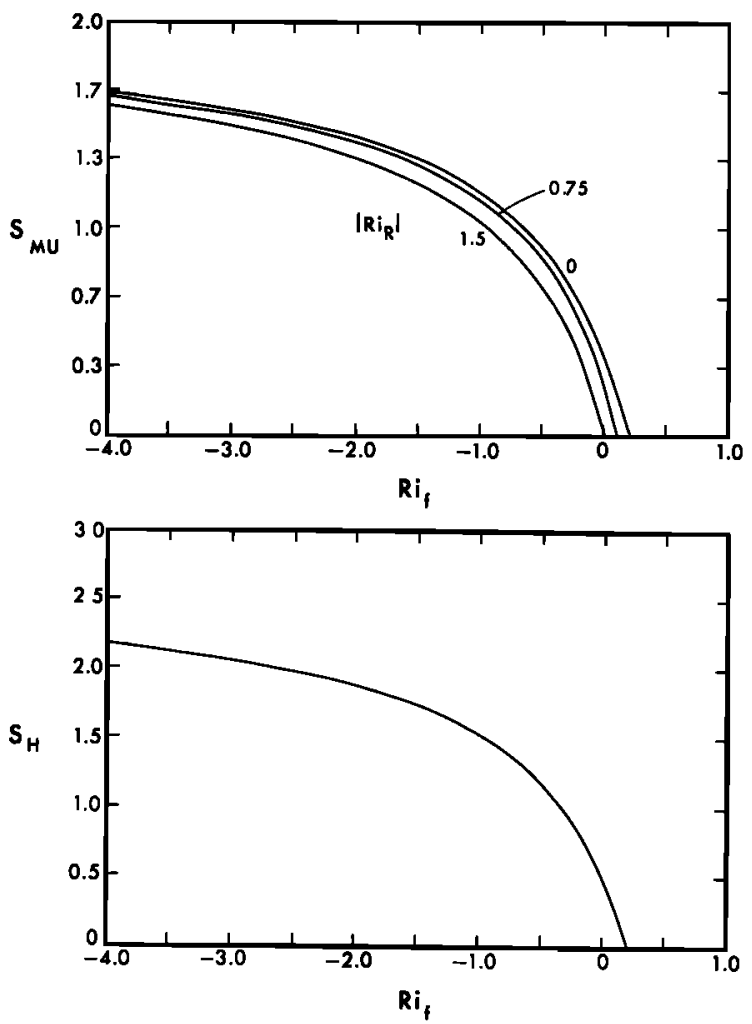

Fig. 1. Plots of mixing coefficients ( $a$ ) for momentum $S_{M}$ and $(b)$ for heat $S_{H}$ as functions of the stratification flux Richardson number $R i$, for various values of rotational Richardson number $R i_{R}$, for the case of nonzero vertical rotation $\left(f \neq 0, f_{v}=0\right)$.

These results can be readily put in the form given by Galperin and Kantha [1989], with $x$ and $y$ axes oriented in the zonal and meridional directions, by a simple coordinate transformation. In the new coordinate system, the Reynolds stresses can be written as

$$
\begin{aligned}
& -\langle\overline{u w}\rangle=q l\left(S_{M U U} \frac{\partial \bar{U}}{\partial z}+S_{M U V} \frac{\partial \bar{V}}{\partial z}\right) \\
& -\left\langle\overline{v w\rangle}=q l\left(S_{M V U} \frac{\partial \bar{U}}{\partial z}+S_{M V V} \frac{\partial \bar{V}}{\partial z}\right)\right.
\end{aligned}
$$

where overbars emphasize the fact that the relevant quantities are in the new coordinate system. It can be shown that the mixing coefficients in (58) are given by

$$
\begin{gathered}
S_{M U U}=S_{M V V}=S_{M U} \frac{(\partial U / \partial z)^{2}}{(\partial U / \partial z)^{2}+(\partial V / \partial z)^{2}} \\
S_{M U V}=-S_{M V U}=S_{M U} \frac{(\partial U / \partial z)(\partial V / \partial z)}{(\partial U / \partial z)^{2}+(\partial V / \partial z)^{2}}
\end{gathered}
$$

and therefore making use of (57), we get

$$
\begin{gathered}
S_{M U U}=B_{1}^{-1 / 3}\left(1+9 A_{1}^{2} R o^{2}\right)^{-1} \\
S_{M U V}=3 A_{1} B_{1}^{-1 / 3} R o^{-1}\left(1+9 A_{1}^{2} R o^{2}\right)^{-1}
\end{gathered}
$$

in agreement with Galperin and Kantha [1989]. Cousteix and Aupoix [1981] have also studied rotating, neutrally stratified fluids, except that their results are valid only for weak rotation. 

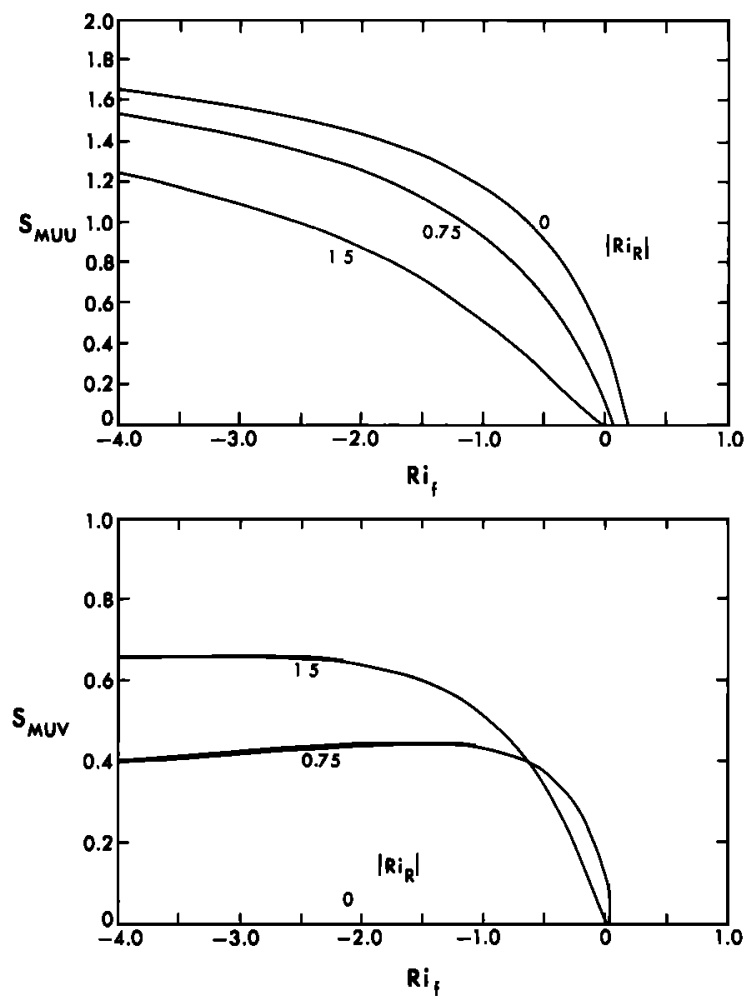

Fig. 2. Plots of tensorial components of momentum mixing coefficients (a) $S_{M U U}$ and (b) $S_{M U V}$ as functions of the stratification flux Richardson number $R i$, for various values of rotational Richardson number $R i_{R}$, for the case of nonzero vertical rotation $(f \neq 0$, $\left.f_{y}=0\right)$.

If we define the rotational Richardson number by

$$
\hat{R} i_{R}=\frac{f}{\left[(\partial U / \partial z)^{2}+(\partial V / \partial z)^{2}\right]^{1 / 2}}
$$

i.e., on the basis of total shear, it can be shown that

$$
\begin{gathered}
S_{M U U}=B_{1}^{-1 / 3}\left(1-9 A_{1}^{2} B_{1}^{-2 / 3} \bar{R} i_{R}^{2}\right) \\
S_{M U V}=3 A_{1} B_{1}^{-2 / 3} \bar{R} i_{R}\left(1-9 A_{1}^{2} B_{1}^{-2 / 3} \bar{R} i_{R}^{2}\right)^{1 / 2}
\end{gathered}
$$

Figure 2 shows $S_{M U U}$ and $S_{M U V}$ plotted as functions of $R i_{f}$ for various values of $\tilde{R} i_{R}$.

\subsection{Constant Flux Region}

In the constant flux region adjacent to a solid surface, (42)-(44) can be written as

$$
\begin{gathered}
\phi_{M}^{c}=\frac{N_{R}+i N_{i}}{D_{R}+i D_{i}}=\phi_{M U}+i \phi_{M V} \\
\phi_{H}=q_{*}^{2}\left[A_{2}\left(1-\frac{6 A_{1}}{B_{1}}\right) q_{*}^{3}-3 A_{2}\left(6 A_{1}+B_{2}\right) \zeta_{M}\right]^{-1} \\
B_{1}\left(\phi_{M U}-\zeta_{M}\right)=q_{*}^{3} \\
N_{R}=\frac{1}{q_{*}}\left[q_{*}^{2}+9 A_{1} A_{2} \zeta_{M} \phi_{H}+9 A_{2}^{2} \zeta_{R}^{2}\right] \\
N_{I}=\frac{3 A_{1} \zeta_{R}}{q_{*}^{2}}\left(q_{*}^{2}-9 A_{2}^{2} \zeta_{M} \phi_{H}+9 A_{2}^{2} \zeta_{R}^{2}\right)
\end{gathered}
$$
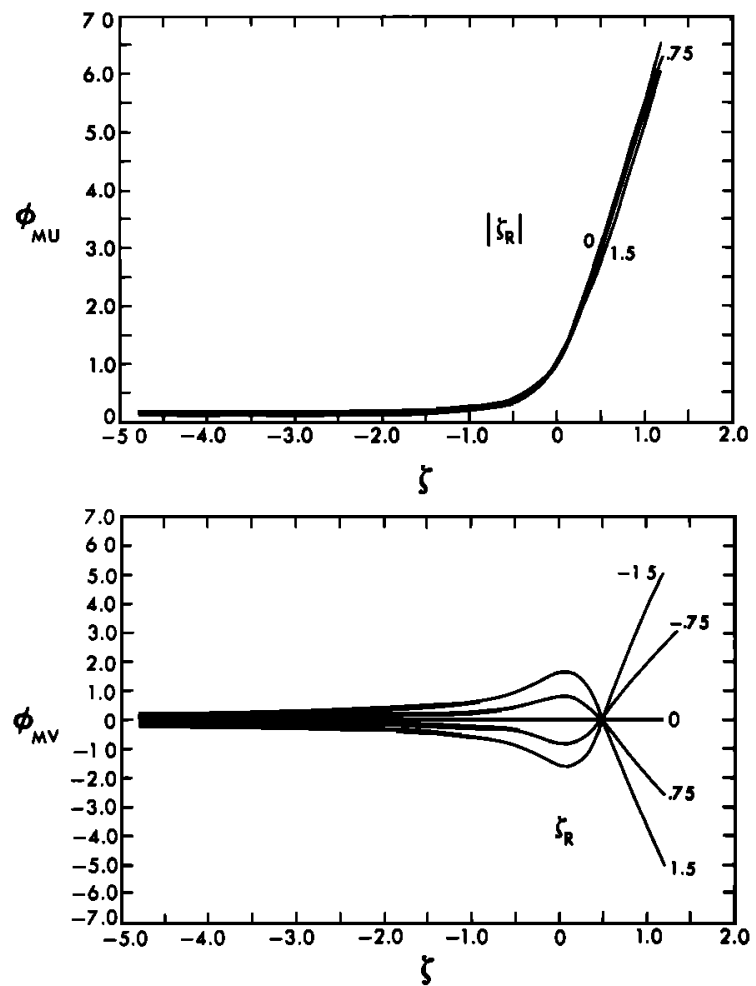

Fig. 3. Plots of Monin-Obukhov similarity functions for (a) the $U$ component of velocity and $(b)$ the $V$ component as functions of $\zeta$, the Monin-Obukhov stratification similarity variable, for various values of the rotational similarity variable $\zeta_{R}$ for the case of nonzero vertical rotation $\left(f \neq 0, f_{v}=0\right)$.

$$
\begin{gathered}
D_{R}=B_{1}^{-1 / 3}\left(q_{*}^{2}+9 A_{2}^{2} \zeta_{R}^{2}\right)-\frac{9 A_{1} \zeta_{M}}{q_{*}^{3}} \\
\cdot\left[\left(2 A_{1}+A_{2}\right) q_{*}^{2}+9 A_{2}^{2} \zeta_{R}^{2}\right] \\
D_{I}=27 A_{1} A_{2}^{2} \zeta_{M} \zeta_{R} / q_{*}^{2}
\end{gathered}
$$

where

$$
\phi_{M U}=\frac{l}{U_{*}} \frac{\partial U}{\partial z}, \phi_{M V}=\frac{l}{U_{*}} \frac{\partial V}{\partial z} \quad \phi_{H}=\frac{l U_{*}}{H} \frac{\partial \Theta}{\partial z}
$$

are Monin-Obukhov type similarity functions for the velocity and temperature profiles, $U_{*}$ is the friction velocity and is equal to $(-\langle u w\rangle)^{1 / 2}$, and $H=-\langle w \theta\rangle$ is the heat flux. The variables $\zeta$ and $\zeta_{R}$ are

$$
\begin{gathered}
\zeta=\frac{l}{L} \\
\zeta_{R}=\frac{l}{L_{R}}
\end{gathered}
$$

where $L=-U_{*}^{3} / \beta g H$ is the stratification Monin-Obukhov length scale and $L_{R}=U_{*} / f$ is an analogous rotation length scale (Ekman scale).

Figures 3 and 4 show $\phi_{M U}, \phi_{M V}$, and $\phi_{H}$, plotted as functions of $\zeta$ for various values of $\zeta_{R}$. The effect of rotation is clearly small, despite the large values used for $\zeta_{R}$.

In the geophysical context, $\zeta_{R}$ seldom exceeds 0.1 or so (see Galperin et al. [1989] for discussion of typical values of 

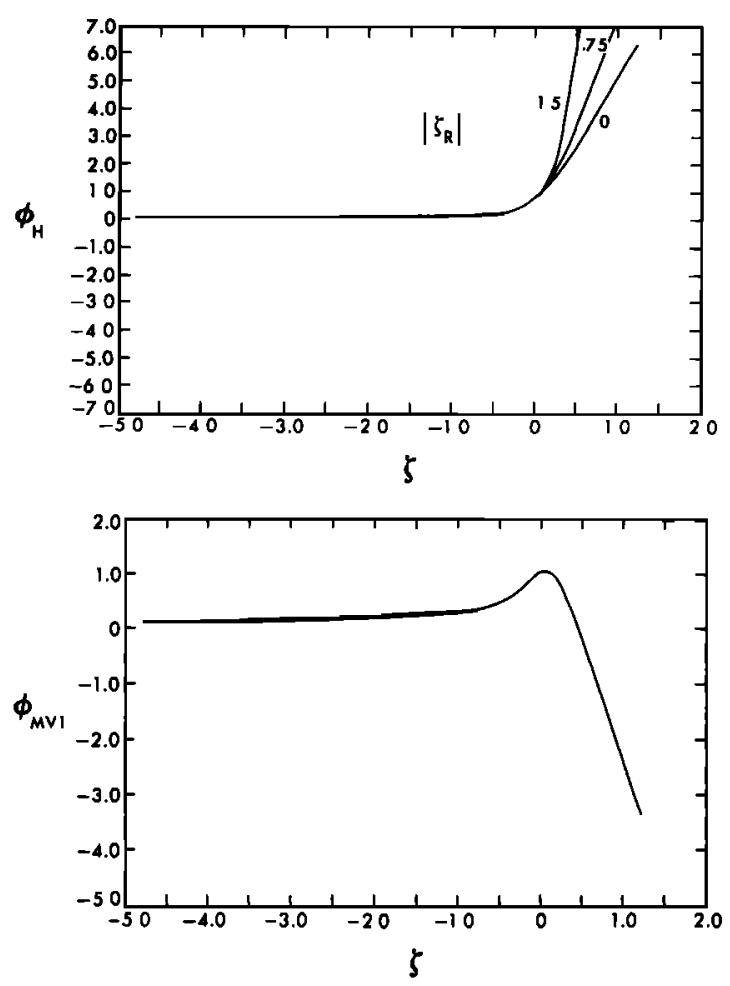

Fig. 4. (a) Plot of Monin-Obukhov similarity functions for temperature as a function of the similarity variable $\zeta$, for various values of the rotational similarity variable $\zeta_{R}$ for the case of nonzero vertical rotation $\left(f \neq 0, f_{v}=0\right)$. (b) Plot of the first-order term $\phi_{M V 1}$ in the expansion $\phi_{M V}=\phi_{M v 0}+\zeta_{R} \phi_{M V 1}$, as a function of $\zeta$, the Monin-Obukhov stratification similarity variable, for the case of nonzero vertical rotation $\left(f \neq 0, f_{v}=0\right)$.

$\zeta_{R}$ ), and therefore small perturbation expansions in terms of $\zeta_{R}$ are in order. It can be shown that the first-order terms are

$$
\phi_{M U 1}=0 \quad \phi_{H 1}=0
$$

but

$$
\begin{aligned}
\phi_{M V 1}=\frac{3 A_{1}\left[q_{* 0}^{2}-9 A_{2}^{2} \zeta \phi_{H 0}\right]}{q_{* 0}^{2}\left[B_{1}^{-1 / 3} q_{* 0}^{2}-9 A_{1} \zeta / q_{* 0}\left(2 A_{1}+A_{2}\right)\right]} \\
\\
-\frac{27 A_{1}^{2} A_{2}^{2} \zeta\left(q_{* 0}^{2}+9 A_{1} A_{2} \zeta \phi_{H 0}\right)}{q_{* 0}^{3}\left[B_{* 1}^{-1 / 3} q_{* 0}^{2}-9 A_{1} \zeta / q_{* 0}\left(2 A_{1}+A_{2}\right)\right]^{2}}
\end{aligned}
$$

where $\phi_{M V}=\phi_{M V 0}+\zeta_{R} \phi_{M V 1}$. Figure $4 b$ shows $\phi_{M V 1}$ plotted as a function of $\zeta$. For neutral stratification,

$$
\phi_{M V 1}=3 A_{1} B_{1}^{-1 / 3}=1.08
$$

When both the stratification and rotation are weak (i.e., $\zeta \ll$ 1 and $\zeta_{R} \ll 1$ ), it is possible to expand in terms of both $\zeta$ and $\zeta_{R}$ to show that

$$
\begin{gathered}
\phi_{M U}=1.0+3.273 \zeta \\
\phi_{M V}=1.08 \zeta_{R} \\
\phi_{H}=0.794+2.755 \zeta
\end{gathered}
$$

\section{Case of Zero Vertical, Nonzero Meridional Rotation $\left[R o=0, R o_{y} \neq 0\right]$}

In the preceding section we considered the case of nonzero $R o$. While interesting, it was apparent that $R o$ does not have a large effect on turbulence, especially at parametric values of interest to geophysics. In this section, we will deal with the geophysically more important case of nonzero meridional rotation $\left(R o_{y} \neq 0\right)$. We will show that $R o_{y}$ can have a large influence on turbulence, although in the geophysical context, the effect is seldom very large. It is worth pointing out that $f_{y}$ does not enter the mean momentum equations, unlike $f$, and therefore its influence on the mean flow is only indirect, through the second-moment equations. In this case, (24) can be written as

$$
\begin{gathered}
S_{M U} \alpha_{u l}\left[1-9 A_{1} A_{2} G_{H}+36 A_{1}^{2} R o_{y}\left(\alpha_{u}+R o_{y}\right)\right] \\
+S_{H}\left[-9 A_{1}\left(2 A_{1}+A_{2}\right) G_{H}\left(\alpha_{u}+R o_{y}\right)\right]=B_{1}^{-1 / 3} \alpha_{u} \\
S_{M U \alpha_{l t}}\left[27 A_{1}^{2} R o_{y} \alpha_{u}\right]+S_{M V} \alpha_{v} \\
\cdot\left[1-9 A_{1} A_{2} G_{H}+9 A_{1}^{2} R o_{y}\left(\alpha_{u}+R o_{y}\right)\right] \\
+S_{H}\left[-9 A_{1}\left(2 A_{1}+A_{2}\right) G_{H} \alpha_{z}\right]=B_{1}^{-1 / 3} \alpha_{v} \\
S_{M U} \alpha_{u l}\left[9 A_{2}\left(2 A_{1}+A_{2}\right) R o_{y}\right]+S_{H}\left[1-3 A_{2}\left(6 A_{1}+B_{2}\right) G_{H}\right. \\
\left.+9 A_{2}^{2} R o_{y}\left(\alpha_{u}+R o_{y}\right)\right]=A_{2}\left(1-\frac{6 A_{1}}{B_{1}}\right)
\end{gathered}
$$

along with the turbulence $\mathrm{KE}$ equation in the limit of local equilibrium:

$$
S_{M U} \alpha_{u}^{2}+S_{M V} \alpha_{v}^{2}+S_{H} G_{H}=B_{1}^{-1}
$$

\subsection{Mixing Coefficients}

Unlike the previous case, the direction of the flow (or equivalently, stress) is important. We will therefore use the conventional coordinate system, with $x$ and $y$ axes aligned in the zonal and meridional directions. Let $\alpha$ be the angle between the shear stress vector and the east, measured positive in the counterclockwise direction. Then if the mixing coefficients and the relevant Richardson numbers are defined by

$$
\begin{gathered}
S_{M U}=\frac{-\langle u w\rangle}{q l(\partial U / \partial z)} \quad S_{M V}=\frac{-\langle v w\rangle}{q l(\partial V / \partial z)} \quad S_{H}=\frac{-\langle w \theta\rangle}{q l \Theta_{z}} \\
R i_{f}=-\beta g\langle w \theta\rangle /\left(-\langle u w\rangle \frac{\partial U}{\partial z}-\langle v w\rangle \frac{\partial V}{\partial z}\right) \\
R i_{R}=f y /\left[\left(\frac{\partial U}{\partial z}\right)^{2}+\left(\frac{\partial V}{\partial z}\right)^{2}\right]^{1 / 2}
\end{gathered}
$$

$S_{M U}, S_{M V}$, and $S_{H}$ become functions of $R i_{f}$ and $R i_{R}$, and equations (74)-(77) can be written as

$\cos \alpha\left\{\left(S_{M U} S_{M V} q_{*}^{4}\right) S_{M U} S_{M V}+36 A_{1}^{2} R i_{R}\left(S_{M V}^{2} \cos ^{2} \alpha\right.\right.$

$$
\begin{aligned}
& \left.+S_{M U}^{2} \sin ^{2} \alpha\right)^{1 / 2} \\
& \cdot\left[S_{M V} \cos \alpha+\left(S_{M V}^{2} \cos ^{2} \alpha+S_{M U}^{2} \sin ^{2} \alpha\right)^{1 / 2} R i_{R}\right] \\
& \left.+9 A_{1} A_{2} R i_{f} \frac{S_{M U} S_{M V}}{S_{H}}\left(S_{M V} \cos ^{2} \alpha+S_{M U} \sin ^{2} \alpha\right)\right\} \\
& +9 A_{1}\left(2 A_{1}+A_{2}\right) R i_{f}\left(S_{M V} \cos ^{2} \alpha+S_{M U} \sin ^{2} \alpha\right)
\end{aligned}
$$

$\left[S_{M V} \cos \alpha+\left(S_{M V}^{2} \cos ^{2} \alpha+S_{M U}^{2} \sin ^{2} \alpha\right)^{1 / 2} R i_{R}\right]$ 
$=B_{1}^{-1 / 3} S_{M V} \cos \alpha\left(S_{M U} S_{M V} q_{*}^{4}\right)$

$$
\begin{aligned}
& 27 A_{1}^{2} R i_{R} S_{M U}\left(S_{M V}^{2} \cos ^{2} \alpha+S_{M U}^{2} \sin ^{2} \alpha\right)^{1 / 2} \cos \alpha \\
& \quad+q_{*}^{4} S_{M U}^{2} S_{M V}^{2}+9 A_{1} A_{2} R i_{f} \frac{S_{M U} S_{M V}}{S_{H}} \\
& \quad \cdot\left(S_{M V} \cos ^{2} \alpha+S_{M U} \sin ^{2} \alpha\right) \\
& \quad+9 A_{1}^{2} R i_{R}\left(S_{M V}^{2} \cos ^{2} \alpha+S_{M U}^{2} \sin ^{2} \alpha\right)^{1 / 2}
\end{aligned}
$$$$
\left[S_{M V} \cos \alpha+R i_{R}\left(S_{M V}^{2} \cos ^{2} \alpha+S_{M U}^{2} \sin ^{2} \alpha\right)^{1 / 2}\right]
$$$$
+9 A_{1}\left(2 A_{1}+A_{2}\right) S_{M U} R i_{f}\left(S_{M V} \cos ^{2} \alpha+S_{M U} \sin ^{2} \alpha\right)
$$$$
=B_{1}^{-1 / 3} S_{M U}\left(S_{M U} S_{M V} q_{*}^{4}\right)
$$

$$
\begin{aligned}
& q_{*}^{4} S_{M U}^{2} S_{M V}^{2}+9 A_{2}\left(2 A_{1}+A_{2}\right) \\
& \cdot \cos \alpha \frac{R i_{R}}{S_{H}} S_{M U} S_{M V}\left(S_{M V}^{2} \cos ^{2} \alpha+S_{M U}^{2} \sin ^{2} \alpha\right)^{1 / 2} \\
&+9 A_{2}^{2} R i_{R}\left[S_{M V}^{2} \cos ^{2} \alpha+S_{M U}^{2} \sin ^{2} \alpha\right]^{1 / 2} \\
& \cdot {\left[S_{M V} \cos \alpha+R i_{R}\left(S_{M V}^{2} \cos ^{2} \alpha+S_{M U}^{2} \sin ^{2} \alpha\right)^{1 / 2}\right] } \\
&+3 A_{2}\left(6 A_{1}+B_{2}\right) R i_{f} \frac{S_{M U} S_{M V}}{S_{H}} \\
& \cdot\left(S_{M V} \cos { }^{2} \alpha+S_{M U} \sin ^{2} \alpha\right) \\
&= A_{2}\left(1-\frac{6 A_{1}}{B_{1}}\right) \frac{S_{M U} S_{M V}}{S_{H}}\left(S_{M U} S_{M V} q_{*}^{4}\right) \\
& q_{*}^{4} S_{M U} S_{M V}=B_{1}\left(1-R i_{f}\right)\left[S_{M U} \sin ^{2} \alpha+S_{M V} \cos ^{2} \alpha\right]
\end{aligned}
$$

These equations can be solved for arbitrary values of $R i_{f}$, $R i_{R}$, and $\alpha$. The solutions are symmetric with respect to the zonal plane; for example, the solution for $\alpha=45^{\circ}$ is the same as that for $\alpha=-45^{\circ}$ if proper allowance is made for the sign of various quantities. We will present results for only the case of zonal flow $\left(\alpha=0^{\circ}\right.$ and $180^{\circ}$ ), of most importance in the geophysical context. In the following plots, positive values of $R i_{R}$ correspond to $\alpha=0^{\circ}$, while negative ones refer to $\alpha=180^{\circ}$.

Figure 5 shows the variation of $S_{M U}$ and $S_{H}$ with $R i_{f}$ for various values of $R i_{R}$. Increasing rotation $\left(R i_{R}\right)$ tends to counteract the effect of unstable stratification and suppress turbulence when $\alpha=0^{\circ}$, while the tendency is exactly the opposite when $\alpha=180^{\circ}$. There is exchange of turbulence KE between the vertical and zonal components with changing $R i_{R}$ (meridional component $\left\langle v^{2}\right\rangle / q^{2}$ does not change), while $q_{*}^{2}$ itself undergoes dramatic changes with $R i_{R}$. Thus it is clear that unlike the case of $f, f_{y}$ can exert a large influence on turbulence. For example, strong stabilizing rotation $\left(R i_{R}\right.$ $>0)$ can suppress turbulence, even when the gravitational stratification is strongly unstable. On the other hand, turbulence can be kept alive for much higher values of stable stratification, by destabilizing rotation $\left(R i_{R}<0\right)$, than would otherwise be feasible. Figure 6 shows the value of critical Richardson number $R i_{f}^{c}$, for which turbulence is quenched, as a function of $R i_{R}$. Outside the domain delineated by the curve, turbulence is extinguished. Thus for neutral flows, turbulence can exist only for $-1.18 \leqslant R i_{R} \leqslant 0.18$ [see
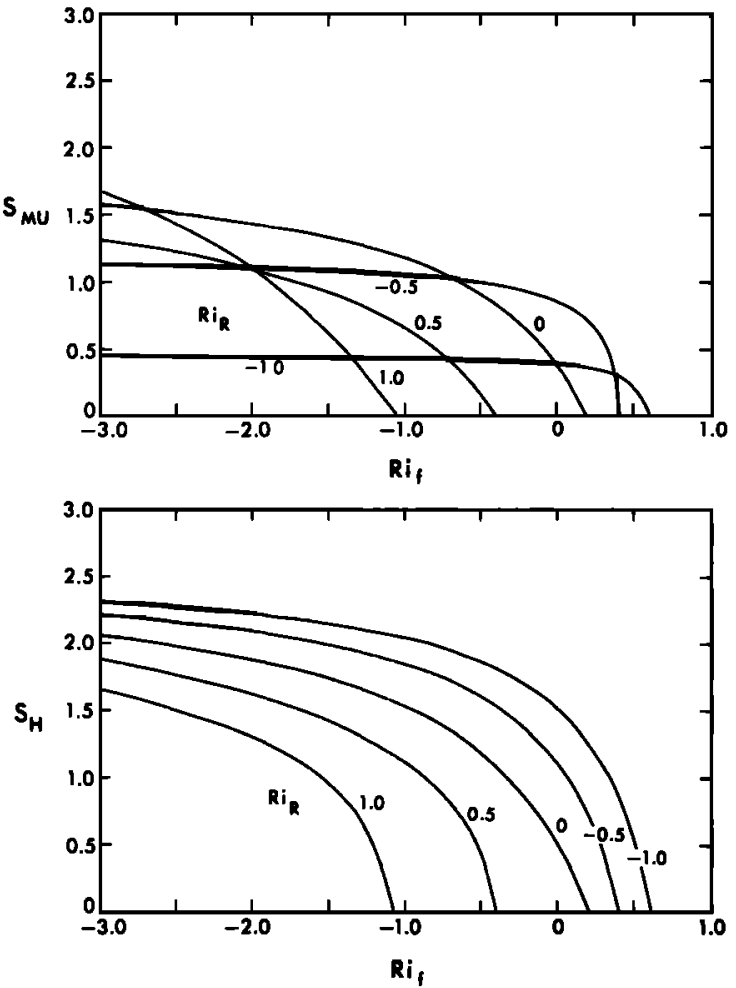

Fig. 5. Same as Figure 1, except for nonzero meridional rotation $\left(f_{y} \neq 0, f=0\right)$.

Galperin et al., 1989; Galperin and Kantha, 1989]. When rotation is destabilizing, turbulence is kept alive at higher stable stratification, up to $R i_{R}=-1$; beyond this value, rotation tends to suppress turbulence and it takes increasingly unstable gravitational stratification to keep turbulence alive, as in the case of stabilizing rotation.

The most important aspect in the geophysical context is the possible range of $R i_{R}$. While strong rotation certainly does have a dramatic influence on turbulence, those values of $R i_{R}$ may not be attainable in practice. In fact, $R i_{R}$ seldom exceeds 0.1 or so for atmospheric and oceanic boundary layers (typical values are $f_{y} \sim 10^{-4} \mathrm{~s}^{-1}, \partial U / \partial z \sim 10^{-3} \mathrm{~s}^{-1}$ ), and therefore while the effect of rotation is nonnegligible, neither is it dramatic. For small values of $R i_{R}$, a small perturbation expansion is appropriate:

$$
\begin{gathered}
S_{M U}\left(R i_{f}, R i_{R}\right)=S_{M U 0}\left(R i_{f}\right)+R i_{R} S_{M U 1}\left(R i_{f}\right)+\cdots \\
S_{H}\left(R i_{f}, R i_{R}\right)=S_{H 0}\left(R i_{f}\right)+R i_{R} S_{H 1}\left(R i_{f}\right)+\cdots
\end{gathered}
$$

Figure 7 shows the variation of the first-order terms $S_{M U 1}$ and $S_{H 1}\left(S_{M V 1}=0\right)$ as a function of $R i_{f}$. The maximum values occur very near neutral stratification. If we take a typical value of 0.1 for $R i_{R}$, it is apparent that rotation can change mixing coefficients by about $30 \%$, a nondramatic but nevertheless a nonnegligible value.

\subsection{Constant Flux Layer}

In the constant flux layer, (24) can be written as

$\cos \alpha\left[q_{*}^{2}+36 A_{1}^{2} \zeta_{R y}\left(\phi_{M U}+\zeta_{R y}\right)+9 A_{1} A_{2} \zeta \phi_{H}\right]$

$$
+9 A_{1}\left(2 A_{1}+A_{2}\right) \zeta\left(\phi_{M U}+\zeta_{R y}\right)=B_{1}^{-1 / 3} \phi_{M U} q_{*}^{3}
$$




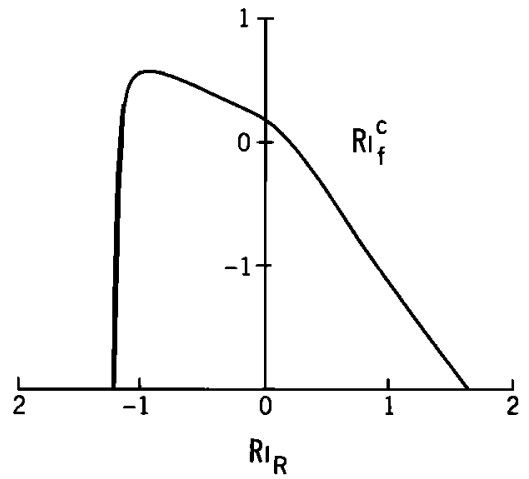

Fig. 6. Critical stratification flux Richardson number $R i_{f}^{c}$ for extinction of turbulence as function of the rotational Richardson number $R i_{R}$ for nonzero meridional rotation $\left(f_{y} \neq 0, f=0\right)$.

$\cos \alpha\left[27 A_{1}^{2} \zeta_{R y} \phi_{M V}\right]+\sin \alpha$

$$
\begin{aligned}
& \cdot\left[q_{*}^{2}+9 A_{1} A_{2} \zeta \phi_{H}+9 A_{1}^{2} \zeta_{R y}\left(\phi_{M U}+\zeta_{R y}\right)\right] \\
& +9 A_{1}\left(2 A_{1}+A_{2}\right) \zeta \phi_{M V}=B_{1}^{-1 / 3} \phi_{M V} q_{*}^{3} \\
q_{*}^{2}+ & \cos \alpha\left[9 A_{2}\left(2 A_{1}+A_{2}\right) \zeta_{R y} \phi_{H}\right]+9 A_{2}^{2} \zeta_{R y}\left(\phi_{M U}+\zeta_{R y}\right) \\
+ & 3 A_{2}\left(6 A_{1}+B_{2}\right) \zeta \phi_{H}=A_{2}\left(1-\frac{6 A_{1}}{B_{1}}\right) \phi_{H} q_{*}^{3}
\end{aligned}
$$

while the turbulence $\mathrm{KE}$ equation in the limit of local equilibrium becomes

$$
q_{*}^{3}=B_{1}\left(\phi_{M U} \cos \alpha+\phi_{M V} \sin \alpha-\zeta\right)
$$

where $\alpha$ denotes the angle that the stress vector makes with respect to the east (positive in the counterclockwise direction). The various nondimensional quantities in (83)-(86) are defined as

$$
\phi_{M U}=\frac{l}{U_{*}} \frac{\partial \mathrm{U}}{\partial \mathrm{z}} \quad \phi_{\mathrm{MV}}=\frac{1}{\mathrm{U}_{*}} \frac{\partial \mathrm{V}}{\partial \mathrm{z}} \quad \phi_{\mathrm{H}}=\frac{\mathrm{I}}{\Theta_{*}} \frac{\partial \Theta}{\partial \mathrm{z}}
$$

where $U_{*}$ is the total friction velocity $\left(U_{*}^{2}=u_{*}^{2}+v_{*}^{2}\right)$ and $\Theta_{*}$ is the friction temperature based on $U_{*}\left(\Theta_{*}=-\langle w \theta\rangle / U_{*}\right)$. The quantities $\zeta$ and $\zeta_{R y}$ are given by

$$
\begin{gathered}
\zeta=\frac{l}{L} \\
\zeta_{R y}=\frac{l}{L_{R y}}
\end{gathered}
$$

and

$$
\begin{gathered}
L=\frac{U_{*}^{3}}{-\beta g\langle w \theta\rangle} \\
L_{R y}=\frac{U_{*}}{f_{y}}
\end{gathered}
$$

are the Monin-Obukhov length scales for stratification and for rotation.

It is possible to solve the set of equations (83) to (86) for any arbitrary value of angle $\alpha$ to obtain $\phi_{M U}, \phi_{M V}$, and $\phi_{H}$ as functions of $\zeta$ and $\zeta_{R y}$. We will present results only for the cases $\alpha=0^{\circ}$ and $180^{\circ}$, which represent cases of zonal flow, of primary importance to geophysical boundary layers.

Figure 8 shows $\phi_{M U}$ and $\phi_{H}\left(\phi_{M V}=0\right)$ as functions of $\zeta$ for various values of $\zeta_{R y}$. Note that positive values of $\zeta_{R y}$ correspond to $\alpha=0^{\circ}$, while the negative values denote $\alpha=$ $180^{\circ}$. The dramatic influence of rotation on Monin-Obukhov similarity functions is clearly seen in these plots.

In the geophysical context, $\zeta_{R y}$ is seldom large, and a small perturbation expansion can be made in terms of $\zeta_{R y}$ for arbitrary values of $\zeta$ :

$$
\begin{gathered}
\phi_{M U}\left(\zeta, \zeta_{R y}\right)=\phi_{M U 0}(\zeta)+\zeta_{R y} \phi_{M U 1}(\zeta)+\cdots \\
\phi_{H}\left(\zeta, \zeta_{R y}\right)=\phi_{H 0}(\zeta)+\zeta_{R y} \phi_{H 1}(\zeta)+\cdots
\end{gathered}
$$

First-order correction terms $\phi_{M U 1}$ and $\phi_{H 1}$ are as shown in Figure 9.

When both stratification and rotational effects are small, it can be shown that

$$
\begin{gathered}
\phi_{M U}\left(\zeta, \zeta_{R}\right)=\cos \alpha\left[1+3.273 \zeta+3.512 \zeta_{R y} \cos \alpha\right] \\
\phi_{M V}\left(\zeta, \zeta_{R}\right)=\sin \alpha\left[1+3.273 \zeta+3.512 \zeta_{R y} \cos \alpha\right] \\
\phi_{H}\left(\zeta, \zeta_{R}\right)=0.794+2.775 \zeta+1.335 \zeta_{R y} \cos \alpha
\end{gathered}
$$

For the zonal case, $\phi_{M V}$ vanishes. The coefficients of $\zeta$ in (90) are well known (see, for example, Mellor [1973] and Galperin et al., [1988]).

Finally, let us note that the relative importance of stratification and meridional rotation in the constant flux region is governed by the ratio

$$
\Pi_{R y}=\frac{\zeta_{R}}{\zeta}=\frac{L}{L_{R y}}
$$

where $L=U_{\star}^{3} / \kappa \beta g H$ is the well-known Monin-Obukhov length scale that indicates the relative magnitude of buoy-
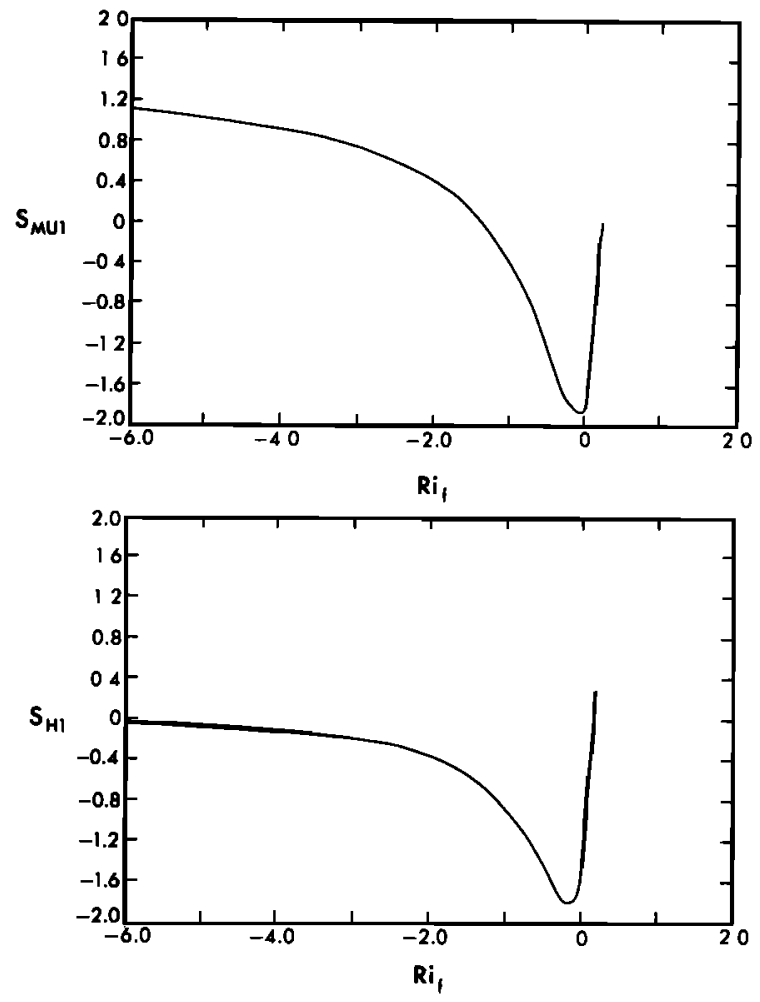

Fig. 7. Plots of the first-order terms (a) $S_{M U 1}$ and (b) $S_{H 1}$ as functions of the stratification flux Richardson number $R i_{f}$ for nonzero meridional rotation $\left(f_{y} \neq 0, f=0\right)$. 

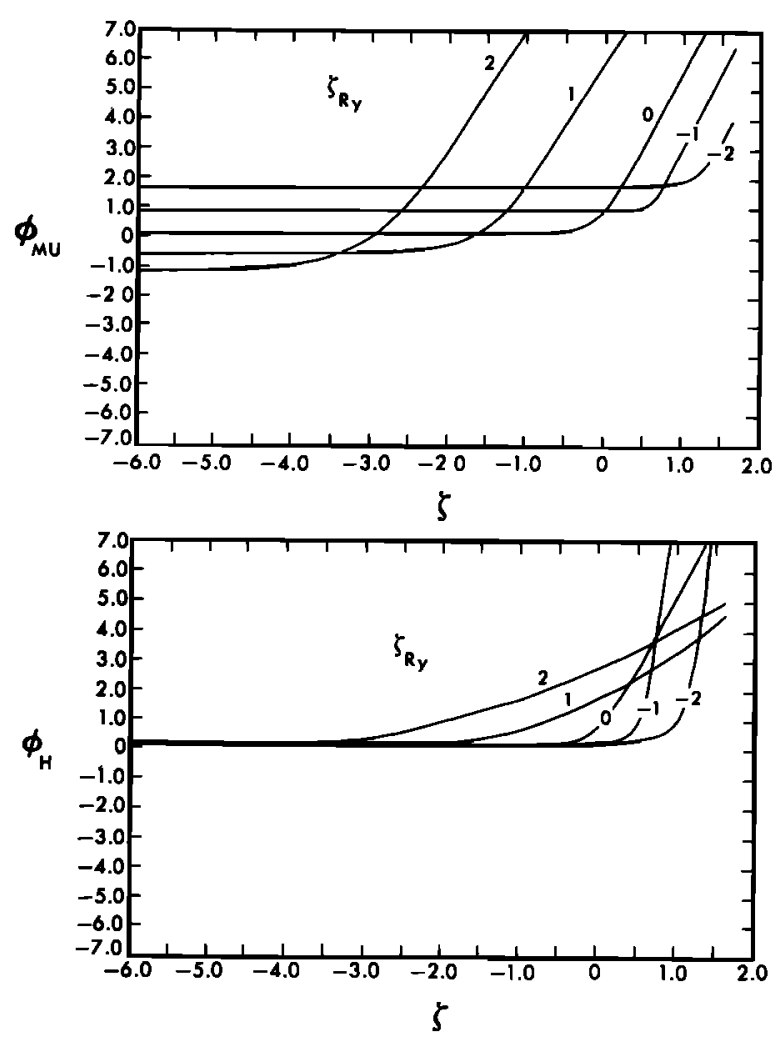

Fig. 8. Plots of Monin-Obukhov similarity functions for $(a)$ velocity $\phi_{M U}$ and (b) temperature $\phi_{H}$ as functions of $\zeta$, the MoninObukhov stratification similarity variable, for various values of the rotational similarity variable $\zeta_{R y}$ for the case of nonzero meridional rotation $\left(f_{y} \neq 0, f=0\right)$.

ancy production vis-à-vis shear production in the surface layer $(H=-\langle w \theta)$, while

$$
L_{R y}=\frac{U_{*}}{f_{y}}
$$

is the corresponding rotation length scale. (For the case of nonzero vertical rotation, $f_{y}$ is replaced by $f$ ).

Equations (24) for the mixing coefficients and their counterparts for the Monin-Obukhov similarity function can in principle be solved for arbitrary values of $R o$ and $R o_{y}$, the two inverse Rossby numbers. However, the algebra is messy, and since in the geophysical context we are interested primarily in the limit of weak rotational effects, we can obtain the necessary solutions from the small perturbation solutions of sections (3) and (4). The small-perturbation solutions for zero $f_{y}$, nonzero $f$ and for zero $f$, nonzero $f_{y}$ can simply be added to produce the desirable solutions for small but nonzero values of both $R o$ and $R o_{y}$.

\section{Concluding Remarks}

In this paper we have given a systematic accounting for the effect of rotation on small-scale turbulence and vertical mixing in stratified fluids, with special emphasis on geophysical flows. A simple turbulence model applicable to a general rotating, stratified flow has been derived using secondmoment closure. By invoking the local equilibrium limit, we have derived simple semianalytical results that provide a better insight into the rotational effects on turbulence in stratified flows. The analysis confirms the hitherto widely
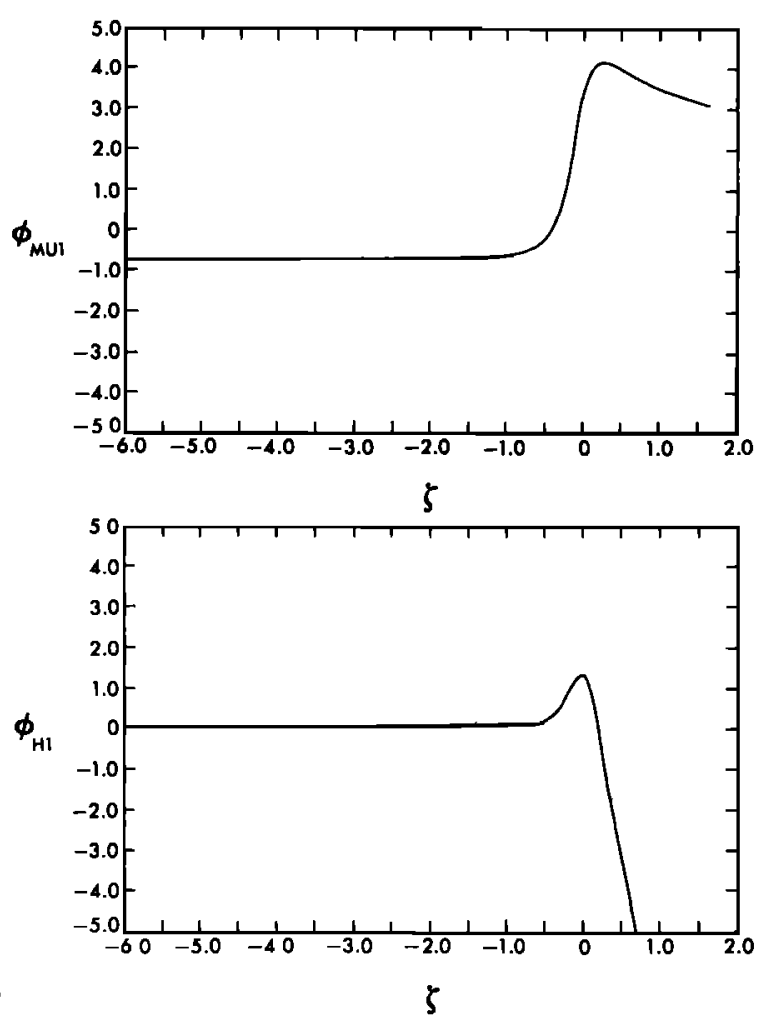

Fig. 9. Plots of first-order terms (a) $\phi_{M U 1}$ and (b) $\phi_{H 1}$ as functions of $\zeta$, the Monin-Obukhov stratification similarity variable, for the case of nonzero meridional rotation $\left(f_{v} \neq 0, f=0\right)$.

held (without rigorous proof, we might add) notion that rotation does not have a profound effect on smallscale turbulence characteristics in geophysical mixed layers. However, the effect on vertical turbulent mixing coefficients can amount to a few tens of percent under certain conditions, clearly nonnegligible though small.

It is clear that strong rotation, especially meridional, can exert a profound influence on turbulence. Laboratory experiments (Johnston et al. [1972] and others) in neutrally stratified flows have shown that turbulence can be strongly enhanced or quenched by strong rotation. The model results are in good agreement with these experiments (see also Galperin and Kantha [1989]). Unfortunately, there are no observations either in the laboratory or in the field that can shed any light on rotational effects on turbulence in stratified fluids. Geophysicists have seldom had to worry about these effects until a recent suggestion by Garwood et al. [1985a, $b$ ]. Although the results of this paper and that of Galperin et al. [1989] suggest that these effects are seldom profound enough to influence geophysical mixed layers drastically, any relevant empirical data that would clarify these aspects further are highly welcome. In addition, rotational effects on turbulent mixed layers might be more dramatic on a more rapidly rotating planetary fluid body.

Acknowledgments. The authors thank Wendy Marshall for doing such a brilliant job in putting this manuscript together. We are greatly impressed by her proficiency and efficiency. We thank Phil Tunison and Jeff Varanyak for their assistance in drafting the figures. L.H.K. thanks the Geophysical Fluid Dynamics Laboratory for providing an atmosphere conducive to this research and acknowledges the support of the Office of Naval Research under grants ONR-N00014-86-C-0438 and ONR-N00014-84-K-0640. 
B.A.G. was supported by the National Science Foundation under grant OCE-8T16027.

\section{REFERENCES}

Bardina, J., J. H. Ferziger, and R. S. Rogallo, Effect of rotation on isotropic turbulence: Computation and modelling, J. Fluid Mech., 154, 321-336, 1985.

Bradshaw, P., Effects of streamline curvature on turbulent flow, AGARDograph 169, 1973.

Cousteix, J., and B. Aupoix, Modélisation des équations aux tensions de Reynolds dans un repère en rotation, Rech. Aerosp., 4, 275-285, 1981.

Galperin, B., and L. H. Kantha, A turbulence model for rotating flows, $A I A A J$., in press, 1989.

Galperin, B., L. H. Kantha, S. Hassid, and A. Rosati, A quasiequilibrium turbulent energy model for geophysical flows, $J$. Atmos. Sci., 45, 55-62, 1988.

Galperin, B., A. Rosati, L. H. Kantha, and G. L. Mellor, Modeling rotating stratified turbulent flows with application to oceanic mixed layers, J. Phys. Oceanogr., in press, 1989.

Garwood, R. W., Jr., P. C. Gallacher, and P. Muller, Wind direction and equilibrium mixed layer depth: General theory, J. Phys. Oceanogr., 15, 1325-1331, 1985a.

Garwood, R. W., Jr., P. Muller, and P. C. Gallacher, Wind direction and equilibrium mixed layer depth in the tropical Pacific Ocean, $J$. Phys. Oceanogr., 15, 1332-1338, 19856.

Hopfinger, E. J., Turbulence in stratified fluids: A review, $J$. Geophys. Res., 92, 5287-5303, 1987.

Hopfinger, E. J., R. W. Griffiths, and M. Mory, The structure of turbulence in homogeneous and stratified rotating fluids, J. Mec: $21-44,1983$.

Johnston, J. P., R. M. Halleen, and D. K. Lezius, Effects of spanwise rotation on the structure of two-dimensional fully developed turbulent channel flow, J. Fluid Mech., 56, 533-557, 1972.

Koyama, H., S. Masuda, I. Abriga, and I. Watanabe, Stabilizing and destabilizing effects of Coriolis force on two-dimensional laminar and turbulent boundary layers, J. Eng. Power, 101, 23, 1979.

Lakshminarayana, B., Turbulence modeling of complex shear flows, AIAA J., 24, 1900-1917, 1986.

Launder, B. E., G. J. Reece, and W. Rodi, Progress in the development of Reynolds-stress turbulence closure, J. Fluid Mech., 68, 537-566, 1975.

Launder, B. E., D. P. Tselepidakis, and B. A. Younis, A second- moment closure study of rotating channel flow, J. Fluid Mech., 183, 63-75, 1987.

Lewellen, W. S., Use of invariant modeling, in Handbook of Turbulence, edited by W. Frost and T. H. Moulden, pp. 237-280, New York, Plenum, 1977.

Mellor, G. L., Analytic prediction of the properties of stratified planetary surface layers, J. Atmos. Sci., 30, 1061-1069, 1973.

Mellor, G. L., and T. Yamada, A hierarchy of turbulence closure models for planetary boundary layers, J. Atmos. Sci., 31, 17911806, 1974. (Correction, J. Atmos. Sci., 34, 1482, 1977).

Mellor, G. L., and T. Yamada, Development of a turbulence closure model for geophysical fluid problems, Rev. Geophys., 20, 851$875,1982$.

Rhines, P. B., Waves and turbulence on a beta-plane, J. Fluid Mech., 69, 417-443, 1975.

Rhines, P. B., The dynamics of unsteady currents, in The Sea, Ideas and Observations on Progress in the Study of the Seas, vol. 6: Marine Modeling, edited by E. D. Goldberg, I. N. McCave, J. J. O'Brien, and J. H. Steele, pp. 189-318, Wiley-Interscience, New York, 1977.

Rhines, P. B., Geostrophic turbulence, Annu. Rev. Fluid Mech., II, $401-441,1979$.

Rodi, W., Examples of calculation methods for flow and mixing in stratified fluids, J. Geophys. Res., 92, 5305-5328, 1987.

Watmuff, J. H., H. T. Witt, and P. N. Joubert, Developing turbulent boundary layers with system rotation, J. Fluid Mech., 157, 405-448, 1985.

Wigeland, R. A., and H. M. Nagib, Grid-generated turbulence with and without rotation about the streamwise direction, Fluids and Heat Transfer Rep. R78-1, Illinois Inst. of Technol., Chicago, 1978.

Zeman, $O$, and $H$. Tennekes, $A$ self-contained model for the pressure terms in turbulent stress equations of the neutral atmospheric boundary layer, J. Atmos. Sci., 32, 1808-1813, 1975.

B. Galperin, Department of Mechanical and Aerospace Engineering, Program in Applied and Computational Mathematics, Princeton University, Princeton, NJ 08544.

L. H. Kantha, Institute for Naval Oceanography, Stennis Space Center, MS 39529.

A. Rosati, Geophysical Fluid Dynamics Laboratory, Princeton, NJ 08542.

(Received July 7, 1988; accepted December 21, 1988.) 